\title{
Probiotic yogurt and acidified milk similarly reduce postprandial inflammation and both alter the gut microbiota of healthy, young men
}

\author{
Kathryn J. Burton ${ }^{1}$, Marta Rosikiewicz ${ }^{2}$, Grégory Pimentel ${ }^{1,3}$, Ueli Bütikofer ${ }^{3}$, Ueli von Ah $^{3}$, \\ Marie-Jeanne Voirol $^{1}$, Antony Croxatto ${ }^{2}$, Sébastien Aeby ${ }^{2}$, Jocelyne Drai ${ }^{4,5}$, Philip G. McTernan ${ }^{6}$, \\ Gilbert Greub $^{2}$, François P. Pralong ${ }^{1}$, Guy Vergères ${ }^{3}$ and Nathalie Vionnet ${ }^{1 *}$ \\ ${ }^{1}$ Service of Endocrinology, Diabetes and Metabolism, Lausanne University Hospital, 1011 Lausanne, Switzerland \\ ${ }^{2}$ Institute of Microbiology, Lausanne University Hospital, 1011 Lausanne, Switzerland \\ ${ }^{3}$ Institute for Food Sciences, Agroscope, Federal Office of Agriculture, 3003 Bern, Switzerland \\ ${ }^{4}$ Laboratoire de Biochimie, Centre Hospitalier Lyon-Sud, 69310 Pierre-Bénite, France \\ ${ }^{5}$ Equipe Inserm CarMeN U1060, Faculté de Médecine Lyon-Sud, BP 12, 69310 Pierre-Bénite, France \\ ${ }^{6}$ School of Science and Technology, Nottingham Trent University, Nottingham NG11 8NS, UK \\ (Submitted 3 February 2017 - Final revision received 21 March 2017 - Accepted 23 March 2017- First published online 31 May 2017)
}

\section{Abstract}

Probiotic yogurt and milk supplemented with probiotics have been investigated for their role in 'low-grade' inflammation but evidence for their efficacy is inconclusive. This study explores the impact of probiotic yogurt on metabolic and inflammatory biomarkers, with a parallel study of gut microbiota dynamics. The randomised cross-over study was conducted in fourteen healthy, young men to test probiotic yogurt compared with milk acidified with $2 \% \mathrm{D}-(+)$-glucono- $\delta$-lactone during a 2 -week intervention $(400 \mathrm{~g} / \mathrm{d})$. Fasting assessments, a high-fat meal test (HFM) and microbiota analyses were used to assess the intervention effects. Baseline assessments for the HFM were carried out after a runin during which normal milk was provided. No significant differences in the inflammatory response to the HFM were observed after probiotic yogurt compared with acidified milk intake; however, both products were associated with significant reductions in the inflammatory response to the HFM compared with the baseline tests (assessed by IL6, TNF $\alpha$ and chemokine ligand 5) $(P<0 \cdot 001)$. These observations were accompanied by significant changes in microbiota taxa, including decreased abundance of Bilophila wadsworthia after acidified milk (log 2-fold-change $\left.(\mathrm{FC})=-1 \cdot 5, P_{\mathrm{adj}}=0.05\right)$ and probiotic yogurt intake $\left(\mathrm{FC}=-1 \cdot 3, P_{\mathrm{adj}}=0.03\right.$ ), increased abundance of Bifidobacterium species after acidified milk intake $\left(\mathrm{FC}=1.4, P_{\mathrm{adj}}=0.04\right)$ and detection of Lactobacillus delbrueckii spp. bulgaricus $\left(\mathrm{FC}=7 \cdot 0, P_{\mathrm{adj}}<0 \cdot 01\right)$ and Streptococcus salivarius spp. thermophilus $\left(\mathrm{FC}=6 \cdot 0, P_{\mathrm{adj}}<0 \cdot 01\right)$ after probiotic yogurt intake. Probiotic yogurt and acidified milk similarly reduce postprandial inflammation that is associated with a HFM while inducing distinct changes in the gut microbiota of healthy men. These observations could be relevant for dietary treatments that target 'low-grade' inflammation.

Key words: Milk: Intestinal microbiota: Inflammation: Probiotics: Prebiotics

The influence of the gut microbiota on health and disease has become increasingly evident over the last decade ${ }^{(1)}$. The evolution in high-throughput sequencing has been critical in enabling the study of the microbiota. Indeed, several large collaborative studies have characterised distinct microbiota patterns relating to disease $\operatorname{risk}^{(2,3)}$. In the context of the increasing prevalence of obesity and type 2 diabetes mellitus, it is striking that gut microbiota composition appears to be different in animals and humans with such diseases, compared with healthy controls ${ }^{(3-5)}$. Thus, the gut microbiota has been identified as an important, potentially modifiable factor that contributes to conditions of metabolic dysfunction.
Dietary modulation of the gut microbiota composition using fermented products containing specific probiotics has been practiced for many years as part of nutritional therapy ${ }^{(6)}$. Defined as live micro-organisms that provide benefits to the host when administered in adequate quantities, ${ }^{(7)}$, probiotics have been extensively studied in relation to health promotion and disease prevention, both as additives to dairy products and as isolated bacterial strains ${ }^{(8)}$. The efficacy of probiotics in promoting health remains disputed, and results differ depending on the outcome of interest, choice of the probiotic strain, formulation of the probiotic and duration of the intervention.

Abbreviations: CCL5, chemokine ligand 5; CFU, colony-forming units; FC, log 2-fold-change; HFM, high-fat meal test; LGG, Lactobacillus rhamnosus GG; LPS, lipopolysaccharide; OTU, operational taxonomic unit.

* Corresponding author: N. Vionnet, fax +41 21692 5595, email Nathalie.Vionnet@chuv.ch 
The use of probiotics to improve metabolic health is a relatively new indication for probiotic treatment. Of particular interest is the potential for probiotics to modulate inflammatory status, as demonstrated both in cell culture ${ }^{(9)}$ and in some human studies $^{(9-11)}$. Chronic, 'low-grade' inflammation is widely recognised as playing a role in the pathological process leading to metabolic disease ${ }^{(12)}$. Furthermore, inflammation in the case of metabolic disorders has been associated with changes of the intestinal microbiota that appear to be in part modulated by the gut-derived factor, lipopolysaccharide (LPS). Indeed, Cani et al. demonstrated that LPS can induce an inflammation that mimics inflammation that is induced by a high-fat meal ${ }^{(13)}$, and proposed that a high-fat diet could contribute to inflammation by increasing the transfer of LPS derived from the gut microbiota across the intestinal barrier ${ }^{(14)}$. The modulation of inflammatory status by dietary intervention, particularly during the early stages of metabolic dysfunction, could thus form a useful part of disease prevention.

Despite the potential for probiotics to modulate metabolic health by gut microbiota-related mechanisms, the gut microbiota is not always assessed in intervention studies that consider the effect of probiotics on metabolic health outcomes ${ }^{(11,15,16)}$, and in cases where analysis is completed, targeted or semitargeted approaches are often adopted ${ }^{(9,10)}$. The development of untargeted metagenomic techniques, such as $16 \mathrm{~S}$ sequencing, offers a more comprehensive approach to understand how the gut microbiota might be influenced by these dietary interventions.

Milk can modulate both the inflammatory response ${ }^{(17)}$ and the composition of the gut microbiota ${ }^{(18)}$. As dairy products are important vectors for the delivery of probiotics to humans, in the present study, we explore the impact of two dairy product dietary interventions on metabolic and inflammatory outcomes, with parallel evaluation of the faecal microbiota dynamics. First, we test the hypothesis that a probiotic yogurt, compared with milk acidified with glucono- $\delta$-lactone, can reduce transient inflammation induced by a high-fat meal challenge. The validated test models a metabolic stimulus which aims to mimic the inflammatory stress that precedes metabolic dysfunction ${ }^{(19)}$. Second, we test the hypothesis that these interventions alter the gut microbial composition.

\section{Methods}

\section{Subjects}

A total of fourteen healthy young men were recruited by a poster campaign (December 2013-March 2014). Inclusion criteria were applied to select subjects aged $18-40$ years with a stable, healthy BMI $\left(18.5-25.0 \mathrm{~kg} / \mathrm{m}^{2}\right)$, regular dietary and physical activity habits, and no evidence of dietary intolerances, restrictions or adverse reactions to dairy products. Exclusion criteria included chronic or acute disease, hypertension, regular medication, moderate or intense physical activity (exceeding $6 \mathrm{~h} /$ week), nutritional supplements, antibiotic treatment in the 6 months preceding the study and a history of anaemia. These criteria were verified during an inclusion visit that included a physical medical examination, dietary and physical activity assessments, standard anthropometrics and bioimpedance analysis (ImpDF50; ImpediMed). Fasting glycaemia, insulinaemia, lipid profile, full blood count and Fe profile were evaluated. This study was conducted according to the guidelines laid down in the Declaration of Helsinki and all procedures involving human subjects were approved by the regional committee for human experimentation (approval no.: 392/13, Vaud, Switzerland). Written informed consent was obtained from all subjects. The trial was registered at clinicaltrial.gov (registration number: NCT02230345).

\section{Interventions}

Two dairy products were tested during the study: yogurt containing the probiotic Lactobacillus rhamnosus GG (LGG) (ATCC $53103)$ and milk acidified with $\mathrm{D}-(+)$-glucono- $\delta$-lactone $(2 \%)$ in order to mimic texture, $\mathrm{pH}$ and physical properties of the yogurt. All dairy products provided during the study were derived from the same batch of full-fat homogenised, ultra-high-temperaturetreated milk (3.5\%) that was supplied by Emmi AG. The probiotic yogurt was prepared by fermentation using Lactobacillus delbrueckii spp. bulgaricus and Streptococcus thermophilus (Thermophilic Yoflex ${ }^{\circledR}$ culture; Chr. Hansen) (online Supplementary Methods). Bacterial counts were carried out for the production batches to confirm a minimum of $1.00 \times 10^{6}$ colonyforming units (CFU)/g per strain: L. delbrueckii spp. bulgaricus $9.04 \times 10^{7} \quad\left(\mathrm{sD} 3.55 \times 10^{7}\right) \mathrm{CFU} / \mathrm{g}, \quad S$. thermophilus $6.50 \times 10^{8}$ $\left(\mathrm{sD} 1.04 \times 10^{8}\right) \mathrm{CFU} / \mathrm{g}$ and LGG $2.83 \times 10^{6}\left(\mathrm{sD} 6.53 \times 10^{5}\right) \mathrm{CFU} / \mathrm{g}$ (online Supplementary Fig. S1). Participants consumed dairy products that were between 4 and $15 \mathrm{~d}$ post-production. The nutritional composition of the products is detailed in the online Supplementary Table S1.

\section{Experimental design}

The experimental design followed the structure of a randomised, double-blind, cross-over trial (Fig. 1(a)). This procedure was used to evaluate the postprandial and short-term effects of the two dairy products investigated, probiotic yogurt and acidified milk. The dairy products were randomly allocated to volunteers (seven volunteers per test sequence). Each product was tested over a 2 -week intervention phase.

Two distinct types of postprandial tests were carried out during the study: (1) dairy product test (D1, D2) and (2) high-fat meal test (HFM) (HFM1, HFM2 and HFM3). The first type of test evaluated the postprandial response to a single dose of the assigned dairy product ( $800 \mathrm{~g}$ of probiotic yogurt or acidified milk). This was conducted on the first day of each test phase. The test marked the beginning of the daily intake phase that required a daily consumption of $400 \mathrm{~g}$ (as per Swiss guidelines for recommended dairy product intake ${ }^{(20)}$ ) of the assigned dairy product for 2 weeks. At the end of this period, a second type of postprandial test was carried out to assess whether the daily intake of probiotic yogurt or acidified milk could influence the response to a meal that normally induces a state of transient inflammation. This test used a mixed-meal challenge (adapted from Schwander et al. $\left.{ }^{(19)}\right)$ formulated to contain an elevated quantity of energy in the form of saturated fat and sugar, and no dairy products (Table 1). The baseline measurements for this 
(a)

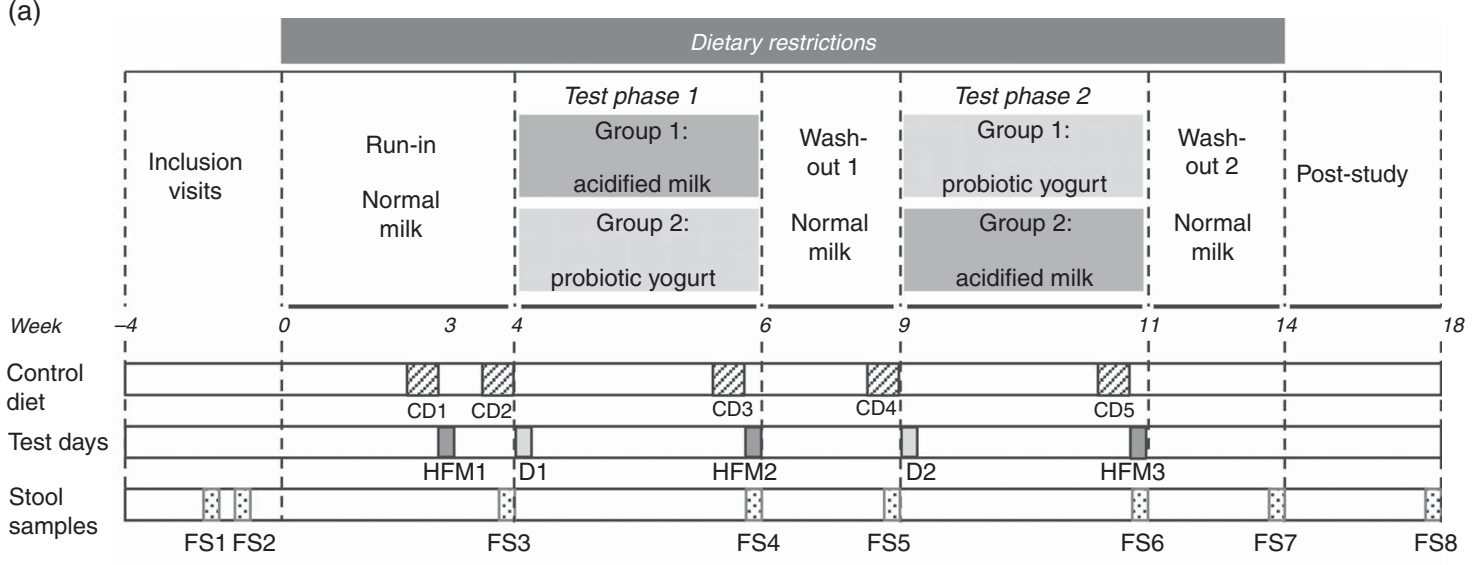

(b)
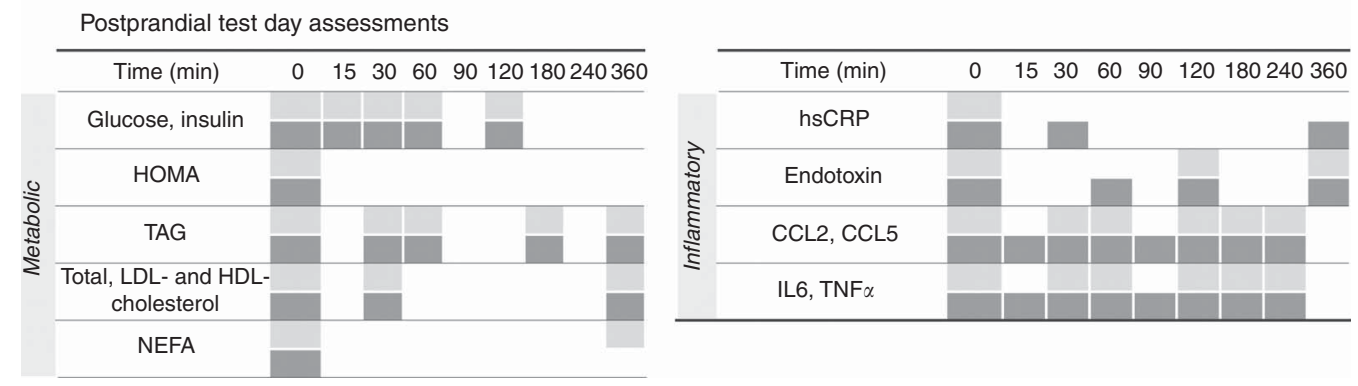

Fig. 1. Overview of the study design. (a) Participants were assigned randomly to group 1 or group 2 to test the probiotic yogurt and acidified milk in a cross-over design. Wash-out periods followed each test phase and a run-in preceded the beginning of the study. (b) Metabolic and inflammatory assessments. $\square$, Evaluations on dairy product test; $\square$, evaluations on high-fat meal test; CD1-5, control diet 1-5; HFM1-3, high-fat meal test days 1-3; D1-2, dairy product test day 1-2; FS1-8, faecal samples 1-8; HOMA, homoeostatic model assessment; hsCRP, high-sensitivity C-reactive protein; CCL2, chemokine ligand 2; CCL5, chemokine ligand 5.

Table 1. Composition of the high-fat meal test (HFM) used to induce postprandial inflammation*

\begin{tabular}{lrrrrrr}
\hline & \multicolumn{6}{c}{ Nutritional content of HFM (per portion) } \\
\cline { 2 - 7 } & Bread & Palm fat & Salami & Eggs & Total & \% Energy \\
\hline Weight (g) & 116.0 & 20.0 & 104.0 & 52.0 & $292 \cdot 0$ & \\
Energy (MJ) & 1.3 & 0.8 & 1.5 & 0.3 & 3.9 & \\
Carbohydrate (g) & 55.7 & 0.0 & 1.0 & 0.2 & 56.9 & 24.8 \\
Sugars (g) & 3.5 & 0.0 & 1.0 & 0.2 & 4.7 & 2.1 \\
Protein (g) & 13.9 & 0.0 & 26.0 & 6.8 & 46.7 & 20.4 \\
Fat (g) & 1.7 & 20.0 & 27.0 & 5.7 & 54.4 & 53.4 \\
SFA (g) & 0.6 & 10.0 & 11.6 & 1.7 & 23.9 & 23.5 \\
MUFA (g) & 0.3 & 8.0 & 12.3 & 2.2 & 22.8 & 22.4 \\
PUFA (g) & 0.8 & 2.0 & 3.1 & 1.0 & 6.9 & 6.7 \\
\hline
\end{tabular}

* Adapted from Schwander et al. ${ }^{(19)}$

test were completed during a run-in period that preceded the first intervention (HFM1 in Fig. 1(a)). During this 4-week run-in period, a fixed dose $(400 \mathrm{ml} / \mathrm{d})$ of full-fat milk was provided to the volunteers to normalise the background dairy product intake before the two dairy product interventions. The same conditions were applied during two 3-week wash-out phases that followed each intervention period.

\section{Dietary and lifestyle restrictions}

Dietary restrictions and assessments were completed during all study phases with additional monitoring of physical activity. Participants were asked to maintain a stable level of physical activity, comparable with their usual pattern of activity, during the study and to avoid intense physical activity during the $3 \mathrm{~d}$ preceding each test day. This was evaluated by ActiGraph wGT3X accelerometers (ActiGraph) worn on the waist during five phases of the study: run-in, test phases $(1,2)$ wash-out phases $(1,2)$. Accelerometers recorded data in $60 \mathrm{~s}$ epochs.

Dietary intake was semi-controlled during the study. Dietary restrictions excluded all dairy products not provided by the study organisers and included specific guidance on portions of fermented foods, alcohol intake and caffeine intake to replicate normal baseline eating patterns. During each test phase of the study, 3-d self-completed dietary records (including 1 weekend day) were completed. In addition, participants self-reported compliance with the assigned dairy product using a daily record. Before each of the five test days, participants followed a 3 -d controlled diet (55\% carbohydrate, 30\% fat, 15\% protein - an example menu is available in the online Supplementary Table S2) (Fig. 1(a), control diet 1-5 (CD1-CD5)). All foods were provided by the study organisers but consumed under free-living conditions. Portions were adapted to meet individual nutritional requirements (estimations completed using the Harris-Benedict equations and physical activity levels 1.5-1.7). Product tolerance and well-being was assessed during each test phase intervention.

\section{Study days}

All five test days were completed at the Centre of Clinical Research, Lausanne, Switzerland. On each test day, the participants 
arrived in a fasted state and a standard clinical assessment that included body weight and composition was carried out (bioimpedance analysis with ImpDF50). A Venflon catheter was placed laterally in the arm of the participant, and after a resting period of $20 \mathrm{~min}$, the first fasting blood sample was taken. The volunteer was then given the test meal, and 6-h postprandial sampling was initiated. A visual analogue scale questionnaire (based on the work of Flint et $a l^{(21)}$ ) was used to assess satiation in the fasted state and postprandially until test completion.

\section{Sampling}

Blood samples were taken at selected time points during the test days (Fig. 1(b)). No blood sampling was done after the completion of wash-out 2. Serum, plasma and whole-blood samples (PAXgene ${ }^{\circledR}$ collection tubes; Qiagen) were prepared according to standard protocols for the selected biomarker. Samples for LPS endotoxin analysis were prepared in sterile tubes $\left(0 \cdot 2 \%\right.$ heparin). All blood samples were stored at $-80^{\circ} \mathrm{C}$.

A total of eight faecal samples were collected from the participants during the study (Fig. 1(a)). These samples were processed under sterile conditions within $4 \mathrm{~h}$ of sample collection. Aliquots $(200 \mathrm{mg}$ ) were added to $2 \mathrm{ml}$ of glycerolbrain heart infusion solution ( $100 \mathrm{ml}$ glycerol, $37 \mathrm{~g}$ Brain Heart Solution, 1 litre distilled water). Following homogenisation by agitation $(13 \boldsymbol{g}, 10 \mathrm{~min})$, samples were stored at $-80^{\circ} \mathrm{C}$. Samples were washed three times with PBS (centrifugation $16000 \mathrm{~g}$, $2 \mathrm{~min}$ ) and suspended at $95^{\circ} \mathrm{C}$ before DNA extraction, which was performed using the QIAamp Fast DNA Stool Mini Kit (Qiagen). Urine samples were collected as a fasted 'spot test' and as a single pooled sample for the $6 \mathrm{~h}$ of each test day using a tube with no additives.

\section{Metabolic and inflammatory marker analyses}

Classical parameters of metabolic health and selected circulating inflammatory markers were analysed at selected time points on the test days (Fig. 1(b)). The COBAS ${ }^{\circledR} 8000$ platform (Roche Diagnostics International AG) was used to assess routine biomarkers (insulin was assessed by the electrochemiluminescence immunoassay assay, all other biomarkers were measured as previously described by van Leckwyck et $\left.a l .{ }^{(22)}\right)$. NEFA were assessed using Wako reagents for NEFA analysis (Wako Diagnostics) on the Pentra 400 platform (ABX; Horiba). Endotoxin levels were assayed using QCL-1000 LAL endpoint assay (Lonza). IL6 and TNF $\alpha$ concentrations were measured by the Bio-Plex Pro ${ }^{\mathrm{TM}}$ Human Cytokine Standard 27-plex, Group 1 assay (BioRad) ${ }^{(23)}$ (intraassay CV $0.52 \%$ for TNF $\alpha, 0.48 \%$ for IL6; inter-assay CV $17 \%$ for IL6, $8 \%$ for TNF $\alpha$ ). Chemokine ligand 2 (CCL2) and chemokine ligand 5 (CCL5) concentrations were measured with the Bio-Plex Pro ${ }^{\mathrm{TM}}$ Human Cancer Biomarker Panel 2, 2-plex assay (BioRad) (intra-assay CV 1.5\% for CCL2, 1.1\% for CCL5; interassay CV 23\% for CCL2, 24\% for CCL5). Both assays were completed using the Luminex ${ }^{\circledR}$ MAGPIX $^{\circledR}$ system (Luminex ${ }^{\circledR}$ Cooperation) that applies magnetic bead methodology. All kits for cytokine and chemokine assessments were from the same kit production batch and all samples from the same volunteer were analysed on the same plate to limit the effect of inter-assay variability. These analyses were performed according to the manufacturer's instructions.

\section{Analysis of faecal microbiota}

DNA libraries were prepared with the extracted faecal DNA using the 16S Metagenomic Sequencing Library protocol, as defined by the Illumina MiSeq System. Primers S-D-Bact-0341-b-S-17 and S-D-Bact-0785-a-A-21 were chosen to target the V3-V4 regions of the bacterial genome ${ }^{(24)}$. Verification of the library quality was completed using the Fragment Analyzer (Advanced Analytical). Sequencing was completed on the Illumina MiSeq and readquality analysis assessed on the Illumina BaseSpace platform.

\section{Nutritional and physical activity analyses}

Accelerometry data were analysed with ActiLife 6 (version 6.10.0). Wear-time validation was completed using the Choi algorithm $^{(25)}$. Data were included in the analysis if the total wear-time for the day assessed exceeded $8 \mathrm{~h}^{(26,27)}$. Outcome parameters were total vector magnitude counts, average vector magnitude counts, counts in light, moderate, vigorous, very vigorous and combined moderate-vigorous activities (Freedson Adult VM3 reference cut-off points ${ }^{(28)}$ ), total number of bouts, total counts during bouts, sedentary bouts, activity energy expenditure, metabolic equivalent of task ${ }^{(29)}$, and weartime.

Food-intake records were analysed by a registered dietitian using the Nutrilog software, version 2.70 with the databases OSAV 5.1 ${ }^{(30)}$, Ciqual $^{(31)}$, Aliments de Marques $^{(32)}$ and manual entry of nutritional data, if available. Total energy intake and macronutrient intake were assessed for all test periods.

\section{Bioinformatic data processing}

The raw paired-reads were assembled into contigs using Pandaseq software ${ }^{(33)}$. Only contigs without any ambigous nucleotides and with a length between 390 and 450 nucleotides were retained in the subsequent analysis. Reference operational taxonomic unit (OTU) sequences were selected using the USEARCH pipeline ${ }^{(34)}$ using the full data set after discarding sequences that were not repeated at least ten times across all samples. Three sequences related to yogurt strains were manually added to the set of OTU reference sequences: Streptococcus salivarius spp. thermophilus, L. delbrueckii spp. lactis and Lactobacillus casei/paracasei. The final abundance of OTU in each sample was counted using the USEARCH package ${ }^{(34)}$.

The 16S rRNA sequences together with taxonomic annotations were downloaded from SILVA database (version 123) ${ }^{(35)}$. All sequences with a pintail quality score ${ }^{(36)}<0.9$ were removed from the database. The reference sequences for OTU were mapped against the database and taxonomy was assigned to the OTU on the basis of best hits, ensuring that the obtained nucleotide similarity exceeded $97 \%$. The species level assignment was kept only in cases of sequences that displayed similarity exceeding $99 \%$. Any sequence that was unassigned using this method was further subjected to classification using online SINA alignment service with default parameters ${ }^{(37)}$. 


\section{Statistical analyses}

The study was designed with two objectives: (1) to explore the changes of metabolomic parameters during postprandial dairy product tests and (2) to study the impact of short-term dairy intake on inflammation. The sample number was chosen to reflect the exploratory nature of the first objective of the study within a highly controlled study design. We report here the results of our second objective. The desired sample number could not be determined because of the absence of previous clinical studies with a similar intervention.

All statistical analyses were performed using $\mathrm{R}$ (version 3.2.4) ${ }^{(38)}$ with applied packages MESS (version $0.3-2)^{(39)}$, DESeq2 (version 1.10.1) ${ }^{(40)}$, phyloseq (version 1.14.0) $)^{(41)}$ and dendextend (version 1.1.8) ${ }^{(42)}$. The Wilcoxon signed-rank test was used to compare the effect of the two dairy product interventions on fasting biomarkers and on the postprandial response to the HFM. Differences were considered significant at $P \leq 0 \cdot 05$. Fasting assessments were evaluated by calculating the respective change after each intervention compared with the baseline levels at the beginning of each test phase. Linear evaluation of the incremental AUC was completed to assess the postprandial response to the HFM (MESS package ${ }^{(39)}$ ). Missing data points for the postprandial response were treated by extrapolation of the postprandial curve where possible, or data were removed if the missing data concerned fasting time points. A pre-test was completed to confirm the assumption of negligible carryover effects, as described by Wellek \& Blettner ${ }^{(43)}$. The treatment effect was then assessed by calculating withinsubject differences for the two sequence groups. The response to the HFM after the dairy product administration was also compared with the baseline response to this test. For this evaluation, the response to each dairy product was compared separately with the baseline test using pairwise comparisons for all subjects pooled, given that all baseline tests preceded the dairy product interventions and no carryover effects were observed. Dietary intake and physical activity changes were assessed using the Kruskal-Wallis rank-sum test using $P \leq 0.05$ to define significant differences.

Microbiota analysis was completed on taxa present at a minimum mean abundance of $0.01 \% /$ volunteer, in at least three volunteers. Cluster analysis was completed at the species level using Spearman's correlation (amap, version 0.8-1.4 $4^{(44)}$ ). DESeq2 was used to complete differential analyses on the microbiota using the Wald test and with significance assessed with $P_{\text {adj }} \leq 0.05^{(40)}$ (Benjamini-Hochberg correction ${ }^{(45)}$ ). The microbiota composition after each test phase was compared directly and analysis was also completed separately for each intervention phase with comparison with pooled samples from all normal-milk phases (run-in, wash-out 1 and wash-out 2). Carryover effects of the intervention were assessed by comparison of samples from wash-out phases after the respective interventions. Time-course changes were assessed by pairwise comparison of each normalmilk phase. In view of the significant inter-individual variation, analysis was completed using samples from volunteers who provided at least one viable faecal sample for each condition being compared in the differential analysis. Diversity indices were assessed by Phyloseq ${ }^{(41)}$, using the Shanon and Simpson indices.

\section{Results}

\section{Participant characteristics}

A total of fourteen healthy young men were enrolled in the study and randomly allocated to one of the two test sequences (group 1: acidified milk-probiotic yogurt; group 2: probiotic yogurt-acidified milk) (online Supplementary Fig. S2). One subject from group 1 was excluded from all analysis because of suspected non-compliance with dietary restrictions that was detected during the microbiota analysis. High levels of the OTU for $L$. case $i$ and $L$. paracase $i$ were detected for this volunteer during the second wash-out phase (faecal sample 7) (online Supplementary Fig. S3(J)). These two bacterial strains are widely used in cheese fermentation and added to dairy products for their probiotic qualities ${ }^{(46,47)}$. The distinct spike in the number of reads apparent for faecal sample 7, together with the absence of the bacteria in all other conditions, is consistent with a discrete intake of a prohibited fermented or probiotic food. Furthermore, negligible counts for the three probiotic yogurt strains were detected for this volunteer after the consumption of the probiotic yogurt (online Supplementary Fig. S3 $(B, G)$ ). The absence of bacterial strains for all other volunteers during run-in, wash-out and acidified milk phases (online Supplementary Fig. S3 (A, C-F, H, I)) indicate compliance with the dairy product dietary restrictions. A second subject (group 2) did not complete the final visit (post-acidified milk) because of acute illness. All the analysis concerning probiotic yogurt was thus completed with twelve participants, whereas the analysis of acidified milk was completed with thirteen participants. Baseline clinical parameters were all within the respective reference ranges (Table 2 ) and showed no difference between the two groups, except for BMI, which was significantly higher for sequence group 1 .

Table 2. Participant characteristics (Medians and interquartile ranges (IQR))

\begin{tabular}{lccc}
\hline & $n$ & Median & IQR \\
\hline Age (years) & 13 & $24 \cdot 0$ & $22 \cdot 0-27 \cdot 0$ \\
Weight (kg) & 13 & $71 \cdot 2$ & $67 \cdot 2-75 \cdot 7$ \\
BMI (kg/m ${ }^{2}$ ) & 13 & $22 \cdot 09$ & $20 \cdot 0-22 \cdot 69$ \\
Fat-free mass (\%) & 13 & $82 \cdot 9$ & $79 \cdot 2-85 \cdot 4$ \\
Fat mass (\%) & 13 & $17 \cdot 1$ & $14 \cdot 6-20 \cdot 8$ \\
Estimated energy requirements (MJ/d) & 13 & $11 \cdot 5$ & $10 \cdot 9-11 \cdot 9$ \\
Estimated protein requirements (g/d) & 13 & $71 \cdot 2$ & $67 \cdot 2-75 \cdot 7$ \\
DBP (mmHg) & 13 & 69 & $67-77$ \\
SBP (mmHg) & 13 & 128 & $120-133$ \\
Heart rate (beats per min) & 13 & 58 & $52-62$ \\
Glycaemia (mmol/l) & 13 & $4 \cdot 7$ & $4 \cdot 6-4 \cdot 9$ \\
Insulin (mU/l) & 13 & $3 \cdot 7$ & $3 \cdot 0-4 \cdot 6$ \\
HOMA score & 13 & $0 \cdot 8$ & $0 \cdot 6-1 \cdot 02$ \\
HbA1c (\%) & 13 & $5 \cdot 0$ & $4 \cdot 8-5 \cdot 3$ \\
TAG (mmol/l) & 13 & $0 \cdot 7$ & $0 \cdot 7-09$ \\
Total cholesterol (mmol/l) & 13 & $3 \cdot 9$ & $3 \cdot 6-4 \cdot 3$ \\
HDL-cholesterol (mmol//) & 13 & $1 \cdot 3$ & $1 \cdot 2-1 \cdot 5$ \\
LDL-cholesterol (mmol/l) & 13 & $2 \cdot 0$ & $1 \cdot 8-2 \cdot 3$ \\
Microbiota biodiversity & & & \\
$\quad$ Shannon Index & 13 & $2 \cdot 9$ & $2 \cdot 79-3 \cdot 22$ \\
Inverse Simpson Index & 13 & $6 \cdot 89$ & $5 \cdot 32-12 \cdot 37$ \\
\hline
\end{tabular}

DBP, diastolic blood pressure; SBP, systolic blood pressure; HOMA, homoeostatic model assessment; HbA1c, glycosylated $\mathrm{Hb}$, type A1c. 


\section{Dietary and physical activity analyses}

Dietary intake during the different phases of the study was not significantly different for macronutrients $(P>0.05)$ (online Supplementary Table S3). In addition, energy expenditure assessed by Actigraph accelerometers showed no significant changes between test phases for sedentary, low, moderatevigorous activity, or for total accelerometer counts by test phase $(P>0.05)$; however, a trend of higher levels of moderatevigorous as well as total counts was observed during the run-in phase (online Supplementary Table S4).

\section{Fasting analyses}

No changes in fasting metabolic and inflammatory markers were observed after 2 weeks' probiotic yogurt intake compared with acidified milk intake, except for CCL2, which showed a greater increase after acidified milk intake $(P=0 \cdot 01)$ (online Supplementary Table S5). The cross-over analysis of the change in fasting markers suggested a difference between the volunteers in sequence groups 1 and 2 for insulin; however, no intervention effect was observed for this marker. In addition, no significant changes were observed for anthropometric measurements or body composition (data not shown).

\section{High-fat meal test response}

No significant differences were observed after probiotic yogurt intake compared with after acidified milk intake for any of the inflammatory and metabolic biomarkers used to assess the postprandial response to the HFM. However, the responses for IL6, CCL5 and TNF $\alpha$ to the HFM were significantly reduced after both interventions in comparison with the baseline evaluations that were completed during the run-in phase $(P<0.001)$ (Fig. 2). A negative outlier was observed for all three biomarkers due to a raised fasting level of inflammation for one volunteer; however, given the use of non-parametric statistical tests, these data did not significantly alter the results. The maximum response for these biomarkers during the baseline (HFM1) test was observed between 90 and $120 \mathrm{~min}$ (online Supplementary Fig. S4). The postprandial response for inflammatory markers high-sensitivity C-reactive protein (hsCRP) and LPS, and metabolic biomarkers were not significantly different after probiotic yogurt or acidified milk intakes, compared with baseline assessments (data not shown).

\section{Microbiota}

Cluster analysis of the microbiota data revealed a strong interindividual variability at the OTU level, with all samples from the same volunteer being clustered together without exception (online Supplementary Fig. S5). Similar grouping was observed at the species and genus levels (data not shown).

The abundance of several bacteria was significantly different after the probiotic yogurt intervention compared with after the acidified milk intervention (online Supplementary Table S6). Notably, the two strains used in the fermentation process, S. salivarius spp. thermophilus and L. delbrueckii spp. bulgaricus, were both significantly more abundant after probiotic yogurt intake (log 2-fold-change $(\mathrm{FC})=7.6$ and $3 \cdot 6$, respectively,
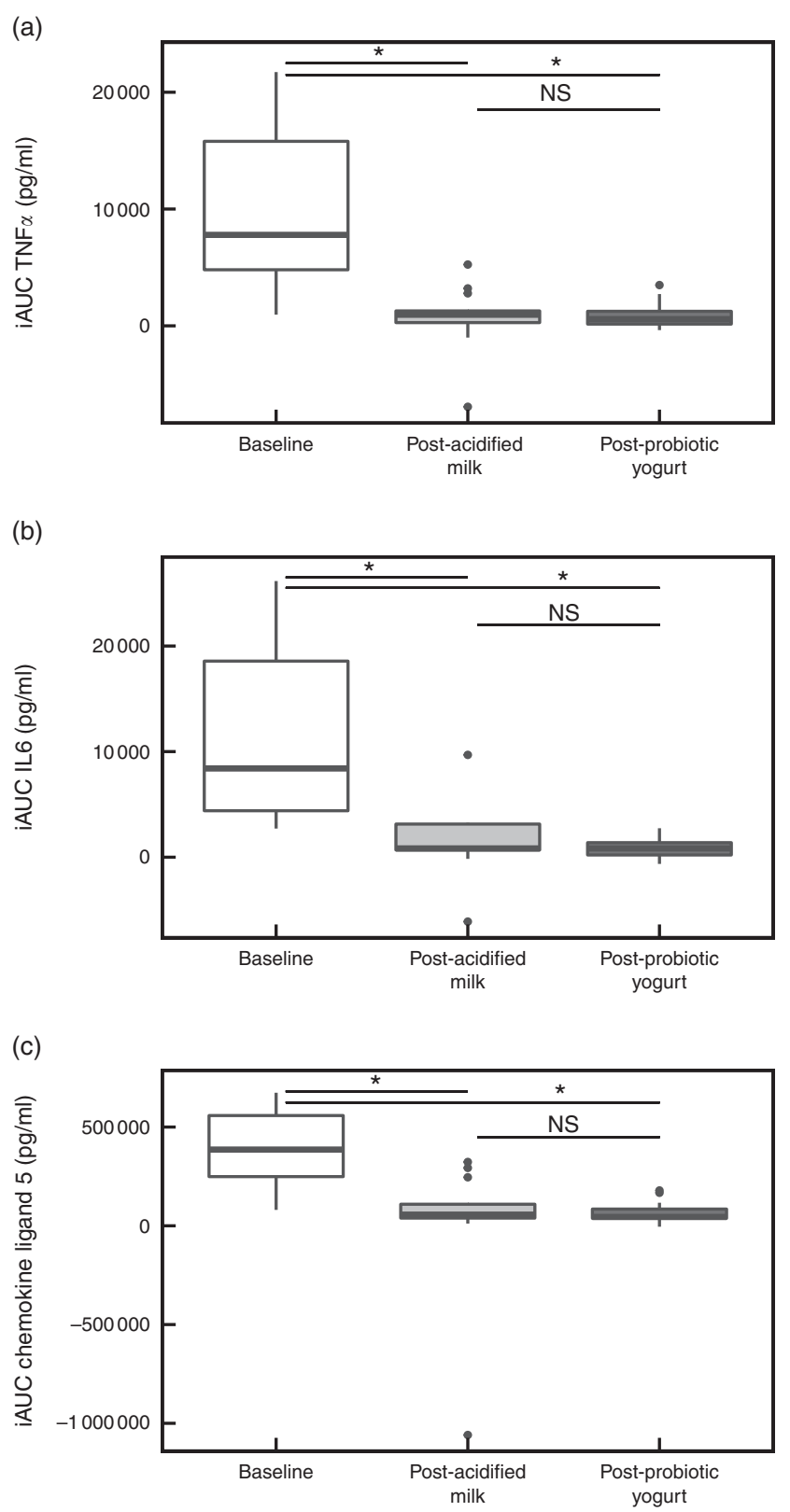

Fig. 2. Postprandial response for inflammatory markers, (a) TNFa, (b) IL6 and (c) chemokine ligand 5 (CCL5), to the high-fat meal test at baseline and after the 2-week intake of acidified milk or probiotic yogurt. iAUC, incremental AUC. ${ }^{\star} P<0.001$.

$\left.P_{\text {adj }}<0 \cdot 01\right)$. The strain Intestinibacter bartlettii was also more abundant after probiotic yogurt intake than after acidified milk intake ( $\left.\mathrm{FC}=2 \cdot 1, P_{\mathrm{adj}}<0 \cdot 01\right)$. Conversely, the Bifidobacterium kashiwanobense or Bifidobacterium pseudocatenulatum strains and the Megasphaera genus were less abundant after probiotic yogurt intake than after acidified milk intake $(\mathrm{FC}=-1 \cdot 7$, $P_{\text {adj }}<0.01$ and $\mathrm{FC}=-1 \cdot 7$, respectively, $P_{\mathrm{adj}}=0.03$ ). These differences were consistently observed at the different taxonomic levels analysed.

Analysis of each intervention phase separately with respect to the baseline (normal milk) phases confirmed the specificity of the differences observed between the interventions (online Supplementary Tables S7 and S8). Transient increases in the 
(a)

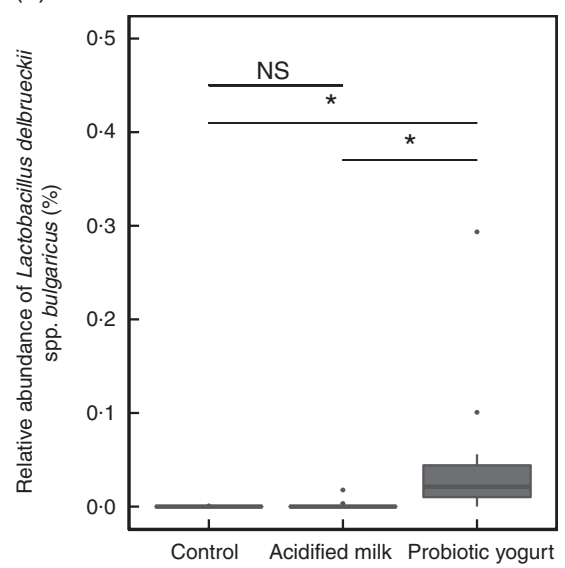

(b)

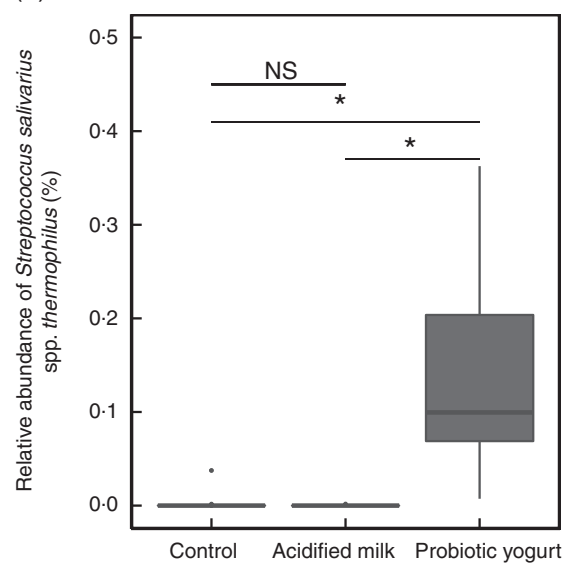

(c)

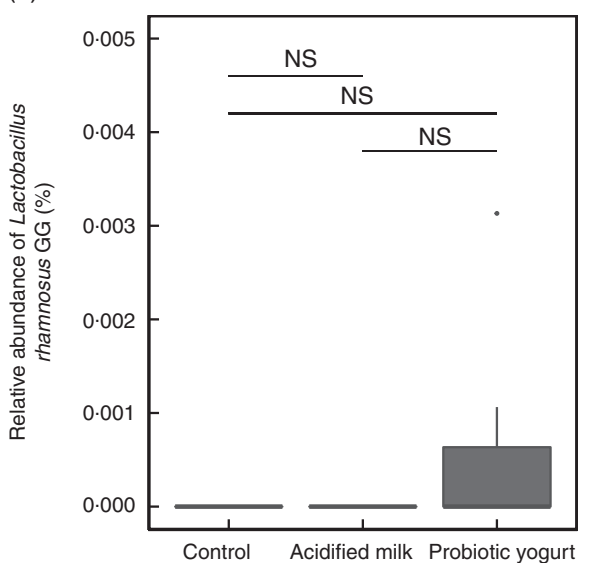

(d)

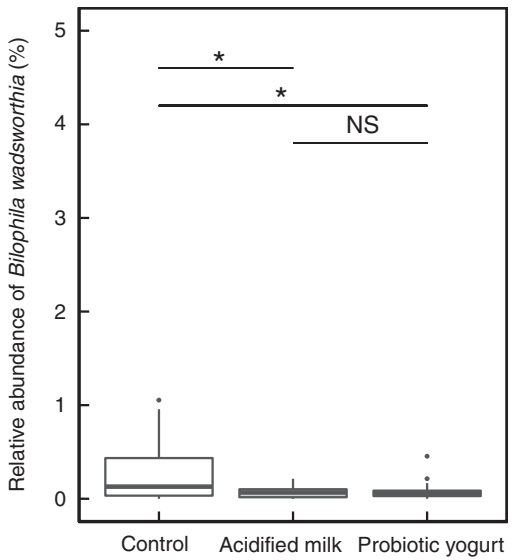

(e)

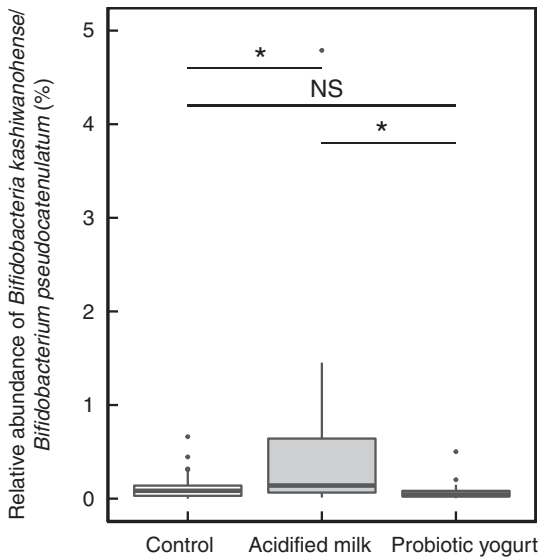

Fig. 3. Changes in selected bacterial species after intake of probiotic yogurt and acidified milk: (a) Lactobacillus delbrueckii spp. bulgaricus; (b) Streptococcus salivarius spp. thermophilus; (c) Lactobacillus rhamnosus GG; (d) Bilophila wadsworthia; (e) Bifidobacteria kashiwanohense/bifidobacteria pseudocatenulatum. Baseline milk (control) compared with interventions acidified milk and probiotic yogurt by differential analysis. ${ }^{*} P_{\text {adj }} \leq 0.05$.

relative abundance of $S$. salivarius spp. thermophilus and L. delbrueckii spp. bulgaricus were observed specifically after probiotic yogurt compared with baseline assessments ( $\mathrm{FC}=6 \cdot 0$ and $7 \cdot 0$, respectively, $\left.P_{\text {adj. }}<0.001\right)$ with no changes in the abundance of these strains after acidified milk intake compared with baseline (Fig. 3(a) and (b)). A non-significant increase in abundance was observed for the probiotic strain LGG after probiotic yogurt intake but not after acidified milk intake (Fig. 3(c)). The difference in abundance of B. kashiwanohense or $B$. pseudocatenulatum between the two interventions was shown to represent, specifically, a relative increase in the strains after acidified milk intake compared with baseline phases (Fig. 3(e)) $\left(\mathrm{FC}=1.4, P_{\mathrm{adj}}=0.04\right)$, with significant effects visible at all taxonomic levels evaluated. These species were not modulated by probiotic yogurt intake compared with baseline phases. The change in abundance of $I$. bartlettii was also associated with the acidified milk intake rather than the probiotic intervention, with significant differences in the abundance of the OTU for the strain after acidified milk intake, compared with baseline assessments ( $\mathrm{FC}=-1 \cdot 7, P=0 \cdot 04)$.

In addition, this analysis revealed further modulation of bacteria by the acidified dairy product interventions.
Notably, significant reductions in abundance for the species Bilophila wadsworthia were observed after both acidified milk and probiotic yogurt intakes, with respect to baseline assessments $\left(\mathrm{FC}=-1.5, P_{\mathrm{adj}}=0.05\right.$ after acidified milk intake; $\mathrm{FC}=-1 \cdot 3, P_{\text {adj }}=0.03$ after probiotic yogurt intake) (Fig. 3(d)). These changes were observed at all levels of taxonomic assessments up to and including class level. Specific changes in the abundance of the species Haemophilus parainfluenzae and six genera were observed after probiotic yogurt intake compared with baseline phases. Acidified milk was associated with relative reductions in the abundance of four OTU of unknown species, and relative increases in abundance of two OTU of unknown species (classified at the genus level as Ruminococcaceae UCG-014 and Lachnospiraceae UCG-004) compared with baseline phases.

Carryover analysis showed no significant changes of any taxa when comparing the wash-out after acidified milk intake with that completed after probiotic yogurt intake (data not shown). However, comparisons of the wash-out phases in study chronological order showed significant changes for the Clostridiaceae Family XIII AD3011 group that was also observed at higher taxonomic classifications from family to class 
level (online Supplementary Table S9)). Differences in bacteria were also observed in comparisons between the run-in phase and the two wash-out phases. Two OTU were different between the run-in and wash-out phase 1, and a further two OTU were different between the run-in and wash-out phase 2 (online Supplementary Tables S10 and S11).

\section{Discussion}

In this cross-over study, probiotic yogurt, compared with acidified milk, did not significantly modulate the postprandial inflammatory response associated with a HFM; however, both products similarly and significantly reduced this inflammatory response, compared with baseline assessments, in our relatively small cohort of healthy young men. These effects were observed in parallel to distinct changes in taxa of the gut microbiota including Lactobacillus, Streptococcus, Bifidobacterium, Bilophila and Ruminococcaceae. The modulation of the microbiota during the acidified milk intervention could be a mechanism for the unexpected effects of this dairy product on the inflammatory response.

We explored postprandial inflammation using a HFM model and observed that daily intake of probiotic yogurt and acidified milk similarly moderate this response. The use of 'challenge' tests such as our HFM can unveil metabolic adaptations that are not detectable in the fasted state. The model simulates physiological exposures to excessive energetic loads and consequentially aims to approach the reality of how the response to dietary excess can be modulated by other dietary components. Although such tests have been used in acute dietary interventions ${ }^{(48,49)}$, few studies have applied the test after a longer exposure to a dietary stimulus ${ }^{(50)}$. Our findings confirm the usefulness of such an approach for evaluating inflammation associated with diet in a healthy population. One of the challenges of evaluating the inflammatory response in clinical studies is the absence of universally accepted biomarkers ${ }^{(51)}$. Using a selection of sensitive blood biomarkers, we detected changes in inflammatory parameters. As in the case of all biomarkers, the clinical significance of these findings needs to be further validated with clinical outcomes. Nevertheless, it was remarkable that we observed a consistent modulation of the IL6, TNF $\alpha$ and CCL5 responses to the HFM by the probiotic yogurt and acidified milk, with respect to baseline tests. Little response to the HFM was observed for hsCRP or LPS both for the baseline tests and tests after the intervention. This may relate to the sensitivity of the markers or could reflect the 'metabolic flexibility' of the healthy volunteers, which seeks to maintain homoeostatic control ${ }^{(19)}$.

The role of dairy products in inflammation is controversial, with evidence for both positive and negative effects described in a recent review of fifty-two clinical trials ${ }^{(17)}$. Overall, a protective role of dairy products on inflammation was suggested in this review, although it was noted that dairy product characteristics and the health status of the population studied influenced this relationship. Given that our baseline challenge test was completed after a run-in period during which normal milk was consumed, it cannot be excluded that the apparent benefits we observe rather represent a relative reduction in inflammation, compared with stimulation of inflammatory parameters by normal milk. Unfortunately, the absence of blood sampling before all milk interventions or following the reintroduction of the participants' normal diets prevented us from testing this hypothesis. Nevertheless, in view of the nutritional qualities of bovine milk, the distinct effects of both probiotic yogurt and acidified milk on inflammation remain an interesting finding.

The concept of modulating the intestinal microbiota in a targeted manner is important in view of the wide number of diseases that are associated with dysbiosis of the intestinal microbiota $^{(52)}$. In the current study, we concur with existing research that demonstrates the highly individual nature of the microbiota composition ${ }^{(53)}$ and the relative stability of this identity over time ${ }^{(54)}$. Indeed, in previous work with salivary microbiota samples, we have already evoked the notion of the microbiota composition as a novel fingerprint which can differentiate individuals ${ }^{(55)}$. The stability of this identity is supported by a recent study that examined the microbiota changes following an antibiotic intervention ${ }^{(56)}$. Despite a rapid perturbation of the microbiota following antibiotic treatment, the inter-individual variability was still largely present following the intervention. Similarly, we observe a significant but distinct modulation of different taxa during the dairy product interventions, whereas the individual identity of the microbiota is maintained. Notably, the acidified milk intervention is associated with a significant increase in Bifidobacterium sp. OTU, which attests to the previously described function of gluconic acid as a prebiotic ingredient ${ }^{(57,58)}$. Whereas gluconic acid has already been used in isolation to induce an increase in this species $^{(57)}$, this is the first study to demonstrate this quality in a dairy product formulation. Bifidobacteria have been associated with multiple health benefits, including benefits on metabolic and inflammatory parameters ${ }^{(15,59)}$. The use of gluconic acid, transformed from glucono- $\delta$-lactone, in combination with a probiotic dairy product, could be an interesting symbiotic approach to maximise the action of the probiotic.

The impact of our probiotic yogurt on the gut microbiota was highly specific to the strains that were present in the product, with limited effect on the commensal microbiota. These findings are in agreement with previous studies that investigated probiotics and in which the gut microbiota was studied ${ }^{(60)}$. The relatively low abundance of LGG in the probiotic yogurt culture explains the absence of significant increases of this strain after the probiotic yogurt test phase, despite the inoculation count exceeding that which has been previously established in dairy products as adequate to ensure faecal recovery of the probiotic $^{(61)}$. One reason for this lower dosage was that at higher inoculation, LGG showed a strong inhibitory effect on the fermenting yogurt strain, L. delbrueckii spp. bulgaricus. Given the beneficial qualities of the yogurt fermenting strains, we opted for a compromise that would favour the growth of the fermenting strains while also meeting minimum levels of LGG to ensure faecal recovery. It should thus be noted that the effects that we observe on inflammation are the combination of a mixed culture including fermenting bacterial strains and LGG. The role of probiotic interventions in metabolic dysfunction is not clear, with an absence of effects reported in some 
studies $^{(11,62,63)}$, although there is some evidence for a role of LGG in reducing inflammation ${ }^{(9,64)}$ and even a report for the role of conventional yogurt in modulating inflammatory markers ${ }^{(10)}$. In our study, we used a relatively low inoculation of LGG, compared with the work of Kekkonen et al ${ }^{(9)}$ in which benefits on fasting inflammatory biomarkers were observed; however, effects on inflammation in healthy populations have been observed at this dosage ${ }^{(64)}$. Further research on the different bacteria used and their relative dosage could provide insight into the positive effects observed on inflammation in our work and may even reveal benefits on fasting markers of inflammation.

A common reduction after acidified milk and probiotic yogurt intakes was observed for the strain B. wadsworthia. The presence of this strain has been associated with acute infections ${ }^{(65)}$, and is modulated by probiotic dairy product interventions ${ }^{(66)}$. Veiga et $a l .{ }^{(67)}$ recently demonstrated that consumption of a fermented milk product decreased the abundance of this opportunistic pathogen in subjects with irritable bowel syndrome. The pro-inflammatory activity of B. wadsworthia in the presence of saturated fat appears to be mediated by its impact on bile salt metabolism and, consequently, lipid uptake ${ }^{(68)}$. Interestingly, $B$. wadsworthia is increased in mice fed a fat-enriched Western diet ${ }^{(69)}$. Our results using full-fat dairy products are in line with evidence in the scientific community to reappraise the impact of dairy foods and milk fat on CVD risk $^{(70)}$.

In our study, metagenomic analysis proved to be a sensitive method that not only showed changes during the interventions but also supported the verification of the compliance of the volunteers with the dairy dietary restrictions. In view of this, we suggest that $16 \mathrm{~S}$ rRNA PCR-based microbiota analysis could be a useful, accessible tool for the evaluation of compliance in probiotic interventions.

One explanation for the association between a high-fat diet, inflammation and the gut microbiota is the transfer of microbiota-derived LPS during lipid uptake ${ }^{(13,14)}$. Whereas we observe a change in bacteria present in the microbiota as well as modulation of inflammatory parameters during our HFM, we were unable to distinguish a significant change in LPS response as persistently low levels of the biomarker were observed on all test days. Given the healthy population studied, it is conceivable that a small change in circulating LPS could lead to larger, measurable changes in circulating interleukins and chemokines. However, these associations may be better elucidated in a population with existing sub-clinical inflammation.

The major strengths of our study are the highly controlled nature of the dietary intervention that included an identical, 3-d controlled diet before each test day. The use of a cross-over design was also an important aspect of the study that facilitated the identification of microbiota changes during the intervention. Given the inter-individual variability in both the baseline microbiota and in the relative changes of the microbiota, use of a parallel study design may not have been adequately sensitive to detect these effects. One unexpected finding in our study was the role of the acidified milk on both the microbiota and the biomarkers of inflammation. This latter effect has not previously been described although there is evidence for the role of other prebiotic foods in modulating inflammation ${ }^{(71)}$. Whereas the study design was not specifically adapted to observe an effect of acidified milk, the baseline tests completed during the run-in phase allowed the effects of the acidified milk to be assessed compared with normal milk. In consideration of the antiinflammatory effects associated with acidified milk, it would be interesting to explore whether the combined use of acidified milk supplemented with probiotic strains could have greater effects of inflammation.

The current study supports a beneficial role of milk supplemented with probiotics and prebiotic ingredients on inflammation associated with a high-fat diet. These benefits may be modulated by modification of the gut microbiota mediated by the probiotic and prebiotic qualities of the tested products. These foods could form a useful part of interventions for populations at risk for inflammation.

\section{Acknowledgements}

The authors wish to thank F. Secretan, C. Pellet, Dr W. Kong and Dr M. van Leckwyck for their clinical assistance and support during the clinical test days; R. Blase and co-workers at Agroscope for the microbiological quality control on the dairy products; and, C. Egger and R. Badertscher for their nutritional analyses of the dairy products.

Endotoxin assays were financed by a collaboration with the University of Warwick using a grant received from the Higher Education Innovation Fund 5. The Higher Education Innovation Fund 5 had no role in the design, analysis or writing of this article.

K. J. B., G. P., A. C., G. G., F. P. P., G. V. and N. V. designed the study; K. J. B., M.-J. V. and N. V. conducted the research; G. P., U. B. and U. v. A. produced and assigned the test products; M. R., K. J. B. and U. B. analysed data and performed statistical analyses; K. J. B., M. R., A. C., U. B., G. G., F. P. P., G. V. and N. V. wrote the paper; N. V. and K. J. B. had primary responsibility for the final content; M.-J. V. completed cytokine assays, P. G. M. completed endotoxin assays, J. D. completed NEFA assays, S. A. completed the microbiota preparation and measurement. All authors read and approved the final manuscript.

None of the authors has any conflict of interest to declare.

\section{Supplementary material}

For supplementary material/s referred to in this article, please visit https://doi.org/10.1017/S0007114517000885

\section{References}

1. Marchesi JR, Adams DH, Fava F, et al. (2016) The gut microbiota and host health: a new clinical frontier. Gut 65, 330-339.

2. Wang Y, Hoenig JD, Malin KJ, et al. (2009) $16 \mathrm{~S}$ rRNA genebased analysis of fecal microbiota from preterm infants with and without necrotizing enterocolitis. ISME J 3, 944-954.

3. Qin J, Li Y, Cai Z, et al. (2012) A metagenome-wide association study of gut microbiota in type 2 diabetes. Nature $\mathbf{4 9 0}$, $55-60$.

4. Ley RE, Backhed F, Turnbaugh P, et al. (2005) Obesity alters gut microbial ecology. Proc Natl Acad Sci U S A 102, 11070-11075. 
5. Ley RE, Turnbaugh PJ, Klein S, et al. (2006) Microbial ecology: human gut microbes associated with obesity. Nature $\mathbf{4 4 4}$ 1022-1023.

6. Collins MD \& Gibson GR (1999) Probiotics, prebiotics, and synbiotics: approaches for modulating the microbial ecology of the gut. Am J Clin Nutr 69, 1052S-1057S

7. Food and Agricultural Organization (FAO) of the United Nations and World Health Organization (WHO) (2006) Health and nutritional properties of probiotics in food including powder milk with live lactic acid bacteria. Report of a Joint FAO/WHO Expert Consultation (2001, Argentina). FAO Food and Nutrition Paper 85. Rome: WHO and FAO. http://www. fao.org/3/a-a0512e.pdf

8. Goldin BR \& Gorbach SL (2008) Clinical indications for probiotics: an overview. Clin Infect Dis 46, Suppl. 2, S96-S100.

9. Kekkonen RA, Lummela N, Karjalainen H, et al. (2008) Probiotic intervention has strain-specific anti-inflammatory effects in healthy adults. World J Gastroenterol 14, 2029-2036.

10. Meyer AL, Elmadfa I, Herbacek I, et al. (2007) Probiotic, as well as conventional yogurt, can enhance the stimulated production of proinflammatory cytokines. J Hum Nutr Diet 20, 590-598.

11. Asemi Z, Samimi M, Tabassi Z, et al. (2013) Effect of daily consumption of probiotic yoghurt on insulin resistance in pregnant women: a randomized controlled trial. Eur J Clin Nutr 67, 71-74.

12. Gonzalez-Chavez A, Elizondo-Argueta S, Gutierrez-Reyes G, et al. (2011) Pathophysiological implications between chronic inflammation and the development of diabetes and obesity. Cir Cir 79, 209-216.

13. Cani PD, Amar J, Iglesias MA, et al. (2007) Metabolic endotoxemia initiates obesity and insulin resistance. Diabetes $\mathbf{5 6}$, 1761-1772.

14. Cani PD, Possemiers S, Van de Wiele T, et al. (2009) Changes in gut microbiota control inflammation in obese mice through a mechanism involving GLP-2-driven improvement of gut permeability. Gut 58, 1091-1103.

15. Bernini LJ, Simao AN, Alfieri DF, et al. (2016) Beneficial effects of Bifidobacterium lactis on lipid profile and cytokines in patients with metabolic syndrome: a randomized trial. Effects of probiotics on metabolic syndrome. Nutrition 32, 716-719.

16. Mohamadshahi M, Veissi M, Haidari F, et al. (2014) Effects of probiotic yogurt consumption on inflammatory biomarkers in patients with type 2 diabetes. Bioimpacts $\mathbf{4}, 83-88$

17. Bordoni A, Danesi F, Dardevet D, et al. (2015) Dairy products and inflammation: a review of the clinical evidence. Crit Rev Food Sci Nutr (epublication ahead of print version 19 August 2015).

18. Charbonneau MR, O'Donnell D, Blanton LV, et al. (2016) Sialylated milk oligosaccharides promote microbiotadependent growth in models of infant undernutrition. Cell 164, 859-871.

19. Schwander F, Kopf-Bolanz KA, Buri C, et al. (2014) A doseresponse strategy reveals differences between normal-weight and obese men in their metabolic and inflammatory responses to a high-fat meal. $J$ Nutr 144, 1517-1523.

20. Infanger E (2004) Table de composition nutritionnelle Suisse, first ed. Bern: Société Suisse de Nutrition (SSN), Office Fédéral de la Santé publique (OFSP), and Ecole polytechnique fédérale (EPF)

21. Flint A, Raben A, Blundell JE, et al. (2000) Reproducibility, power and validity of visual analogue scales in assessment of appetite sensations in single test meal studies. Int J Obes Relat Metab Disord 24, 38-48.
22. van Leckwyck M, Kong W, Burton KJ, et al. (2016) Decreasing insulin sensitivity in women induces alterations in LH pulsatility. J Clin Endocrinol Metab 101, 3240-3249.

23. Schmid A, Petry N, Walther B, et al. (2015) Inflammatory and metabolic responses to high-fat meals with and without dairy products in men. BrJ Nutr 113, 1853-1861.

24. Klindworth A, Pruesse E, Schweer T, et al. (2013) Evaluation of general $16 \mathrm{~S}$ ribosomal RNA gene PCR primers for classical and next-generation sequencing-based diversity studies. Nucleic Acids Res $\mathbf{4 1}$, e1.

25. Choi L, Liu Z, Matthews CE, et al. (2011) Validation of accelerometer wear and nonwear time classification algorithm. Med Sci Sports Exerc 43, 357-364.

26. Evenson KR \& Terry JW Jr (2009) Assessment of differing definitions of accelerometer nonwear time. Res $Q$ Exerc Sport 80, 355-362

27. Chinapaw MJ, de Niet M, Verloigne M, et al. (2014) From sedentary time to sedentary patterns: accelerometer data reduction decisions in youth. PLOS ONE 9, e111205.

28. Freedson PS, Melanson E \& Sirard J (1998) Calibration of the Computer Science and Applications, Inc. accelerometer. Med Sci Sports Exerc 30, 777-781.

29. Sasaki JE, John D \& Freedson PS (2011) Validation and comparison of ActiGraph activity monitors. J Sci Med Sport 14, 411-416.

30. OSAV (Office Fédéral de la Sécurité Alimentaire et des Affaires Vétérinaires) (2015) Base de données Suisse des Valeurs Nutritives. Version 5.1. http://naehrwertdaten.ch/

31. ANSES (Agence nationale de sécurité sanitaire de l'alimentation, de l'environnement et du travail) (2013) Ciqual French food composition table version 2013. https://pro.anses.fr/ TableCIQUAL/

32. Blanc JP (2003) Table française de composition d'aliments industriels. In Le Guide de la Minceur, first ed. Paris: Editions First.

33. Masella AP, Bartram AK, Truszkowski JM, et al. (2012) PANDAseq: paired-end assembler for illumina sequences. BMC Bioinformatics 13, 31 .

34. Edgar RC (2013) UPARSE: highly accurate OTU sequences from microbial amplicon reads. Nat Methods 10, 996-998.

35. Quast C, Pruesse E, Yilmaz P, et al. (2013) The SILVA ribosomal RNA gene database project: improved data processing and web-based tools. Nucleic Acids Res $\mathbf{4 1}$, D590-D596.

36. Ashelford KE, Chuzhanova NA, Fry JC, et al. (2005) At least 1 in 20 16S rRNA sequence records currently held in public repositories is estimated to contain substantial anomalies. Appl Environ Microbiol 71, 7724-7736.

37. Pruesse E, Peplies J \& Glockner FO (2012) SINA: accurate high-throughput multiple sequence alignment of ribosomal RNA genes. Bioinformatics 28, 1823-1829.

38. R Core Team (2016) $R$ : A Language and Environment for Statistical Computing. Vienna: R Foundation for Statistical Computing.

39. Ekstrøm C (2014) MESS: Miscellaneous Esoteric Statistical Scripts. R package version 0.3-2. https://CRAN.R-project.org/ package $=$ MESS

40. Love MI, Huber W \& Anders S (2014) Moderated estimation of fold change and dispersion for RNA-seq data with DESeq2. Genome Biol 15, 550.

41. McMurdie PJ \& Holmes S (2013) phyloseq: an R package for reproducible interactive analysis and graphics of microbiome census data. PLOS ONE 8, e61217.

42. Galili T (2015) dendextend: an R package for visualizing, adjusting and comparing trees of hierarchical clustering. Bioinformatics 31, 3718-3720. 
43. Wellek S \& Blettner M (2012) On the proper use of the crossover design in clinical trials: part 18 of a series on evaluation of scientific publications. Dtsch Arztebl Int 109, 276-281.

44. Lucas A (2014) amap: Another Multidimensional Analysis Package. R package version 0.8-14. https:/CRAN.R-project. org/package $=$ amap

45. Benjamini Y \& Hochberg Y (1995) Controlling the false discovery rate: a practical and powerful approach to multiple testing. J R Statist Soc Series B 57, 289-300.

46. Carafa I, Nardin T, Larcher R, et al. (2015) Identification and characterization of wild lactobacilli and pediococci from spontaneously fermented Mountain cheese. Food Microbiol 48, 123-132.

47. Holzapfel WH, Haberer P, Geisen R, et al. (2001) Taxonomy and important features of probiotic microorganisms in food and nutrition. Am J Clin Nutr 73, 365S-373S.

48. Edirisinghe I, Banaszewski K, Cappozzo J, et al. (2011) Strawberry anthocyanin and its association with postprandial inflammation and insulin. Br J Nutr 106, 913-922.

49. Burton-Freeman B, Talbot J, Park E, et al. (2012) Protective activity of processed tomato products on postprandial oxidation and inflammation: a clinical trial in healthy weight men and women. Mol Nutr Food Res 56, 622-631.

50. Ellis CL, Edirisinghe I, Kappagoda T, et al. (2011) Attenuation of meal-induced inflammatory and thrombotic responses in overweight men and women after 6-week daily strawberry (Fragaria) intake. A randomized placebo-controlled trial. J Atheroscler Thromb 18, 318-327.

51. Calder PC, Ahluwalia N, Albers R, et al. (2013) A consideration of biomarkers to be used for evaluation of inflammation in human nutritional studies. Br J Nutr 109, Suppl. 1, S1-S34.

52. Carding S, Verbeke K, Vipond DT, et al. (2015) Dysbiosis of the gut microbiota in disease. Microb Ecol Health Dis 26, 26191.

53. Turnbaugh PJ, Hamady M, Yatsunenko T, et al. (2009) A core gut microbiome in obese and lean twins. Nature $\mathbf{4 5 7}$, 480-484.

54. Faith JJ, Guruge JL, Charbonneau M, et al. (2013) The longterm stability of the human gut microbiota. Science $\mathbf{3 4 1}$, 1237439.

55. Leake SL, Pagni M, Falquet L, et al. (2016) The salivary microbiome for differentiating individuals: proof of principle. Microbes Infect 18, 399-405.

56. Dethlefsen L \& Relman DA (2011) Incomplete recovery and individualized responses of the human distal gut microbiota to repeated antibiotic perturbation. Proc Natl Acad Sci US A 108, Suppl. 1, 4554-4561.
57. Asano T, Yuasa K, Kunugita K, et al. (1994) Effects of gluconic acid on human faecal bacteria. Microbial Ecol Health Dis 7, 247-256.

58. Tsukahara T, Koyama H, Okada M, et al. (2002) Stimulation of butyrate production by gluconic acid in batch culture of pig cecal digesta and identification of butyrate-producing bacteria. J Nutr 132, 2229-2234.

59. Meng H, Ba Z, Lee Y, et al. (2015) Consumption of Bifidobacterium animalis subsp. lactis BB-12 in yogurt reduced expression of TLR-2 on peripheral blood-derived monocytes and pro-inflammatory cytokine secretion in young adults. Eur J Nutr 2, 649-661.

60. Eloe-Fadrosh EA, Brady A, Crabtree J, et al. (2015) Functional dynamics of the gut microbiome in elderly people during probiotic consumption. MBio 6, e00231-15.

61. Saxelin M (1996) Colonization of the human gastrointestinal tract by probiotic bacteria. Nutr Today 31, Suppl. 1, 5S-8S.

62. Gobel RJ, Larsen N, Jakobsen M, et al. (2012) Probiotics to adolescents with obesity: effects on inflammation and metabolic syndrome. J Pediatr Gastroenterol Nutr 55, 673-678.

63. Mazloom Z, Hejazi N \& Dabbaghmanesh MH (2009) Effects of obesity on inflammation and lipid profile of obese women. Saudi Med J 30, 1357-1358.

64. Schultz M, Linde HJ, Lehn N, et al. (2003) Immunomodulatory consequences of oral administration of Lactobacillus rhamnosus strain GG in healthy volunteers. J Dairy Res 70, 165-173.

65. Finegold S, Summanen P, Hunt Gerardo S, et al. (1992) Clinical importance of Bilophila wadsworthia. Eur J Clin Microbiol Infect Dis 11, 1058-1063.

66. Odamaki T, Kato K, Sugahara H, et al. (2016) Effect of probiotic yoghurt on animal-based diet-induced change in gut microbiota: an open, randomised, parallel-group study. Benef Microbes 7, 473-484.

67. Veiga P, Pons N, Agrawal A, et al. (2014) Changes of the human gut microbiome induced by a fermented milk product. Sci Rep 4, 6328.

68. Devkota S \& Chang EB (2015) Interactions between diet, bile aid metabolism, gut microbiota, and inflammatory bowel diseases. Dig Dis 33, 351-356.

69. Devkota S, Wang Y, Musch MW, et al. (2012) Dietary-fatinduced taurocholic acid promotes pathobiont expansion and colitis in Il10-/- mice. Nature 487, 104-108.

70. German JB, Gibson RA, Krauss RM, et al. (2009) A reappraisal of the impact of dairy foods and milk fat on cardiovascular disease risk. Eur J Nutr 48, 191-203.

71. Lomax AR \& Calder PC (2009) Prebiotics, immune function, infection and inflammation: a review of the evidence. $\mathrm{BrJ}$ Nutr 101, 633-658. 